
allemande

51-1 | 2019

La République démocratique allemande dans l'espace public européen (1949-2018)

\title{
La peinture, médium privilégié de l'art contemporain allemand
}

Hélène Trespeuch

\section{(2) OpenEdition}

\section{Journals}

Édition électronique

URL : https://journals.openedition.org/allemagne/1636

DOI : $10.4000 /$ allemagne. 1636

ISSN : 2605-7913

Éditeur

Société d'études allemandes

Édition imprimée

Date de publication : 2 juillet 2019

Pagination : 247-257

ISSN : 0035-0974

Référence électronique

Hélène Trespeuch, «La peinture, médium privilégié de l'art contemporain allemand », Revue d'Allemagne et des pays de langue allemande [En ligne], 51-1 | 2019, mis en ligne le 02 juillet 2020,

consulté le 02 juin 2022. URL : http://journals.openedition.org/allemagne/1636 ; DOI : https://doi.org/ 10.4000/allemagne.1636 


\title{
La peinture, médium privilégié de l'art contemporain allemand
}

\author{
- Hélène Trespeuch *
}

\section{Un lien privilégié entre la peinture contemporaine et l'Allemagne}

Depuis les années 1990, l'art contemporain s'ouvre de plus en plus à des artistes venus d'Asie, du Moyen-Orient, d'Afrique ou d'Amérique du Sud. Si ces effets de la mondialisation sont visibles, notamment dans la programmation des institutions muséales, il n'en demeure pas moins que les États-Unis et l'Allemagne demeurent les deux principaux pays prescripteurs en matière d'art contemporain. Pour s'en convaincre, il suffit de jeter un œil sur les résultats du «Kunstkompass» des dernières années. Dans ce classement des artistes vivants les plus renommés ${ }^{(1)}$ - qui a le mérite de ne pas prendre en considération les données marchandes de l'art (prix des œuvres, résultats des ventes aux enchères, etc.) - il est aisé de constater que les nationalités états-uniennes et allemandes sont les mieux représentées, et ce depuis plusieurs décennies ${ }^{(2)}$. En octobre 2011, l'historien de l'art et critique Philippe Dagen interrogeait cette domination de l'art allemand dans un article publié dans Le Monde sous le titre suivant: «L'art allemand est supérieur». Afin d'expliquer la place de choix occupée par le pays sur l'échiquier international du monde de l'art, l'article mettait en avant la stimulante structure fédérale, le soutien patriotique à la création émanant de

* Maître de conférences en histoire de l'art contemporain, Université Paul-Valéry Montpellier 3.

1 Le classement du "Kunstkompass» prend en compte les expositions individuelles consacrées à des artistes vivants dans un certain nombre de musées internationaux jugés importants, la participation de ces artistes à des expositions collectives dans ces mêmes institutions, ainsi que les articles qui leur seraient consacrés dans certaines revues ou journaux influents du monde de l'art. Ainsi, ce classement s'intéresse tout particulièrement à l'aura médiatique des artistes vivants. Il existe depuis 1970 et paraît chaque année dans un magazine allemand. En 2018, il s'agissait du magazine Capital.

2 Voir les analyses du sociologue Alain Quemin, notamment Les stars de l'art contemporain. Notoriété et consécration artistiques dans les arts visuels, Paris, CNRS Éditions, 2013. Voir également les résultats du « Kunstkompass» 2018 brièvement esquissés à la page suivante: www.zeit.de/news/2018-11/22/ kunstkompass-gerhard-richter-wichtigster-kuenstler-181122-99-916338. 
puissants collectionneurs, ainsi que l'excellent niveau des Kunstakademien. Philippe Dagen notait en outre que «l'une des forces de l'art allemand est sa diversité de modes et de styles", rappelant qu'au cours des cinq dernières décennies, l'Allemagne s'est autant distinguée avec des peintres (Gerhard Richter, Sigmar Polke, Martin Kippenberger, Albert Oehlen, etc.) qu'avec des artistes familiers de la performance comme Beuys ou Vostell (le premier concert du groupe Fluxus a eu lieu à Wiesbaden), des artistes conceptuels comme Hans Haacke, ou encore des photographes comme ceux de la célèbre école de Düsseldorf (Thomas Ruff, Thomas Struth, Andreas Gursky, Candida Höfer, etc.). À ce propos, l'historien de l'art allemand Werner Spies, un des quatre experts interrogés dans cet article, remarquait toutefois que «les peintres sont toujours restés forts, y compris dans les années 1960 et $1970 »^{(3)}$.

Ce point mérite quelques tentatives d'explication. Que l'Allemagne ait des atouts qui lui permettent de bien défendre ses artistes est un fait indéniable et relativement bien connu. Il peut sembler néanmoins plus surprenant de constater que les artistes allemands les plus renommés - si l'on se fie, du moins, au «Kunstkompass » - se trouvent être systématiquement des peintres. En effet, depuis des années caracolent en tête de ce classement les peintres Gerhard Richter (à la première place depuis 15 ans), Georg Baselitz, Anselm Kiefer, et jusqu'à sa mort en 2010, Sigmar Polke - ainsi que Rosemarie Trockel, mais en tant qu'artiste conceptuelle, et non peintre. Il apparaît donc que, malgré la diversité des modes d'expression artistique qui s'observe dans l'art contemporain allemand, les artistes les plus renommés sont avant tout des peintres, célébrés à la fois par les institutions muséales internationales et la presse spécialisée.

Ce constat pourrait paraître anodin; cependant, dans la sélection très réduite des artistes vivants les plus réputés, ces artistes allemands sont quasiment les seuls à avoir jeté leur dévolu sur le médium peinture, les artistes d'autres nationalités - leurs directs concurrents dans ce classement - s'intéressant majoritairement à d'autres moyens d'expression, tels que la performance, l'installation, la vidéo, la photographie. Les artistes américains les plus renommés par exemple, tels que Bruce Nauman, Cindy Sherman ou Richard Serra, travaillent la vidéo, la photographie, la sculpture, et non la peinture. Cette observation autorise à penser, non pas que seule l'Allemagne abriterait sur son territoire des peintres de qualité, mais plutôt qu'il existerait un lien privilégié entre la peinture et la scène artistique allemande. Autrement dit, les peintres pourraient bénéficier d'un soutien solide et durable en Allemagne, ce qui ne serait peut-être pas le cas aux États-Unis ou dans les autres pays d'Europe s'imposant dans le «Kunstkompass ». Lorsqu'en 2011, Werner Spies déclarait que «les peintres sont toujours restés forts» en Allemagne, précisant "y compris dans les années 1960 et 1970 », il renvoyait très vraisemblablement à la situation observée aux États-Unis au cours de ces décennies. En effet, alors que s'y développaient l'art conceptuel, le Land art ou encore le Body art, la peinture était un médium rejeté par une large partie de la scène artistique nationale. Lui étaient préférées des formes d'expression plus difficilement commercialisables, comme la performance, l'installation éphémère, la vidéo, qui éprouvaient également la logique de conservation des musées. Un virulent discours anti-peinture accompagnait

3 Werner Spies, cité dans Philippe DAgen, «L'art allemand est supérieur», Le Monde, $1^{\text {er }}$ octobre 2011, p. 3. 
ces développements artistiques aux États-Unis, diffusant l'idée selon laquelle la peinture serait devenue un médium obsolète, affichant une dangereuse complicité avec le marché, et par là même avec toute l'idéologie capitaliste ${ }^{(4)}$. Il reste à comprendre comment et pourquoi l'Allemagne a résisté à ce désaveu de la peinture qui émanait à l'époque du principal pays prescripteur en matière d'art contemporain, à savoir les États-Unis.

\section{Plusieurs générations de peintres allemands à la renommée internationale}

Il importe tout d'abord de souligner que si quelques peintres allemands (Gerhard Richter, Georg Baselitz, Anselm Kiefer) figurent parmi les artistes les plus renommés du «Kunstkompass», ils sont loin d'être les seuls à bénéficier d'une reconnaissance internationale. En effet, plusieurs générations de peintres coexistent aujourd'hui en Allemagne, bénéficiant d'un intérêt qui dépasse les frontières germaniques. Cette continuité contribue indéniablement à la puissance et la grande visibilité de la peinture allemande contemporaine sur la scène occidentale puisque cette situation génère le sentiment que ce pays est un terreau extraordinaire pour la peinture contemporaine. Le lien privilégié entre l'Allemagne et la peinture n'est donc pas propre à un petit groupe de peintres, il est plus structurel que circonstanciel.

La plus ancienne génération de peintres allemands contemporains est ainsi constituée par des artistes qui commencent leur carrière dans les années 1960. Il s'agit notamment de Gerhard Richter (1932) et de Sigmar Polke (1941-2010) - qui se font connaître en 1963 au travers de leurs expositions-performances annonçant un nouveau mouvement qu'ils situent dans la filiation du Pop art américain, mais qu'ils inscrivent dans un contexte bien allemand en l'intitulant «Réalisme capitaliste». À cette même génération appartiennent Georg Baselitz (1938), qui fait scandale la même année 1963 avec son œuvre Große Nacht im Eimer (la scène de masturbation est jugée immorale et l'œuvre saisie par la justice), ou encore A.R. Penck (1939) et Markus Lüpertz $^{(5)}$ (1941). La deuxième génération est constituée par des artistes qui se font connaître dans les années 1970, notamment Anselm Kiefer ${ }^{(6)}$ (1945), Jörg Immendorf (1945-2007), Imi Knoebel (1940) ou Blinky Palermo (1943-1977) - qui ont tous eu pour enseignant Joseph Beuys à la Kunstakademie de Düsseldorf. Ensuite, les années 1980 sont indéniablement associées au regroupement médiatique des «Nouveaux

4 Voir par exemple Douglas CRIMP, «The End of Painting», October, printemps 1981, p. 69-86. Pour une analyse approfondie, voir Hélène Trespeuch, La Crise de l'art abstrait? Récits et critique en France et aux États-Unis dans les années 1980, Rennes, Presses universitaires de Rennes, 2013, p. 35 sqq.

5 Tous ces peintres ont bénéficié récemment d'expositions rétrospectives de leur œuvre organisées ou accueillies en France: Gerhard Richter au Centre Pompidou à Paris en 2012, Sigmar Polke au musée de Grenoble en 2014, Georg Baselitz au musée Unterlinden à Colmar en 2018, et au musée d'art moderne de la ville de Paris en 2011, A.R. Penck au musée d'art moderne de la ville de Paris en 2008, et dans ce même musée, Markus Lüpertz en 2015.

6 Les peintres de cette génération ont également présenté leurs œuvres lors d'expositions personnelles dans les musées français: Anselm Kiefer a bénéficié d’une rétrospective au Centre Pompidou, à Paris, en 2016; une importante exposition des œuvres d’Imi Knoebel a eu lieu au musée de Grenoble en 1996; Palermo en 1985 au Centre Pompidou à Paris. Les œuvres de Jörg Immendorf ont été présentées au Centre Pompidou en 1993, mais cette exposition a duré moins d'un mois. 
fauves", ce label confus attribué aux peintres allemands associés au phénomène de «retour à la peinture» alors observé sur toute la scène contemporaine occidentale ${ }^{(7)}$. De ces années subsiste aujourd'hui, en termes de reconnaissance internationale, le nom de quelques artistes, à l'instar de Martin Kippenberger (1953-1997) ou Albert Oehlen $^{(8)}$ (1954). La décennie suivante voit apparaître Neo Rauch ${ }^{(9)}$ (1960) ou encore Daniel Richter ${ }^{(10)}$ (1962), quand les années 2000 accueillent Jonathan Meese (1971), mais aussi les artistes de l' «école de Dresde» avec Ebehard Havekost (1967), Thomas Scheibitz (1968) ou encore Frank Nitsche ${ }^{(11)}$ (1964), ainsi que ceux de la «nouvelle école de Leipzig», aux côtés de Neo Rauch (Tim Eitel, Matthias Weischer, David Schnell ${ }^{(12)}$, etc.). Cette remarquable succession et coexistence sur la scène allemande de peintres d'horizons différents génèrent le sentiment que ce pays a su devenir un terreau extraordinaire pour la peinture contemporaine.

Tous ces peintres ont naturellement des styles très différents, des conceptions de la peinture (et de l'art en général) parfois divergentes. Certains sont nés en RDA, quand d'autres n'ont connu que la RFA. La majorité d'entre eux ont une production majoritairement figurative, mais, sur les bases avancées par Gerhard Richter et Sigmar Polke, qui ont réalisé des tableaux aussi bien figuratifs qu'abstraits, ce constat se révèle peu pertinent. En effet, à la suite de leurs illustres aînés, beaucoup de peintres allemands depuis les années 1970 questionnent l'image au sens large - comme Albert Oehlen ou Ebehard Havekost -, et non le registre trop cloisonné de la figuration ou de l'abstraction. En outre, ce serait oublier les contributions non négligeables de quelques Allemands à l'histoire de l'abstraction contemporaine, comme Helmut Dorner (1952), Katharina Grosse (1961), Franz Ackermann (1963) ou encore Tomma Abts ${ }^{(13)}$ (1967). Les différentes orientations plastiques de tous ces artistes allemands ne permettent donc pas de penser qu'il y ait un style, un mode d'expression spécifique qui aurait été valorisé plus qu'une autre au sein de la peinture allemande contemporaine.

7 Cette appellation «retour à la peinture» n'avait en réalité de justification qu'aux États-Unis où la peinture avait été rejetée dans les années 1960-1970. En effet, cette situation ne s'observait pas avec la même radicalité dans les autres pays de la scène occidentale au cours de ces décennies.

8 Albert Oehlen a présenté ses œuvres en France lors d'expositions personnelles au musée d'art moderne et contemporain de Strasbourg en 2003, au FRAC Auvergne à Clermont-Ferrand en 2005, au musée d'art moderne de la ville de Paris en 2009, ainsi qu'au Carré d'art de Nîmes en 2011. Une rétrospective de l'œuvre de Martin Kippenberger a été organisée en 2008 par le Museum of contemporary art de Los Angeles.

9 Aucune exposition personnelle de Neo Rauch n'a été présentée en France. Toutefois, le musée Frieder Burda de Baden-Baden a publié en 2011 un catalogue d'exposition en français.

10 Hors des frontières allemandes, Daniel Richter a bénéficié d’une importante exposition en 2006 au Museum für Gegenwartskunst de Bâle.

11 À l'exception de Thomas Scheibitz, les trois autres artistes ont bénéficié d'une exposition personnelle au FRAC Auvergne à Clermont-Ferrand: Jonathan Meese en 2005, Frank Nitsche en 2007 et Ebehard Havekost en 2008.

12 Seul Tim Eitel a présenté ses œuvres dans une institution française: en 2004 au CRAC Alsace à Altkirch.

13 Parmi ces artistes, deux ont présenté leurs œuvres lors d'expositions individuelles au FRAC Auvergne à Clermont-Ferrand: Helmut Dorner en 2000 (qui avait également exposé au Carré d’art de Nîmes en 1991), Katharina Grosse en 2007. Le FRAC Champagne-Ardenne à Reims a organisé une exposition sur l'œuvre de Franz Ackermann en 2005. 


\section{Du désintérêt des années 1970 à la gloire des années 1980}

Comment donc comprendre cette place singulière et manifestement privilégiée de la peinture dans l'art contemporain allemand? L'étude d'ouvrages généraux et de catalogues d'exposition sur l'art contemporain allemand autorise à supposer que la peinture a été le mode d'expression plastique ayant permis à l'Allemagne (dans un premier temps, à la RFA) de s'affirmer comme une scène artistique puissante, capable de concurrencer les États-Unis. La peinture aurait été valorisée et bien défendue sur le sol allemand, précisément parce que d'autres pays de la scène internationale la bannissaient.

Cette situation ne s'observe toutefois pas encore dans les années 1970. Au cours de cette décennie, les peintres qui apparaissent aujourd'hui comme les fers de lance de la création germanique sont alors peu mis en valeur. Dans l'ouvrage du critique et artiste allemand Rolf-Gunter Dienst intitulé Deutsche Kunst, eine neue Generation, datant de 1970, les productions de Sigmar Polke, Georg Baselitz ou encore Markus Lüpertz sont en effet absentes ${ }^{(14)}$. Seules les œuvres de Gerhard Richter sont intégrées à ce panorama de l'art contemporain allemand, aux côtés d'artistes n'ayant pas (ou peu) développé de pratique picturale, comme les artistes associés à Fluxus tels que Joseph Beuys et Wolf Vostell, et ceux liés à l'art conceptuel comme Hans Haacke. En dehors de Gerhard Richter, d'autres peintres sont pourtant présentés, mais leur production fait surtout écho aux grandes tendances internationales ( $p o p$ art, minimal art, art optique et cinétique) et paraît ainsi moins singulière que celle des peintres allemands précédemment cités. D’ailleurs, l'œuvre de Gerhard Richter est présentée sous la double bannière de l'hyperréalisme et du Pop art. En 1973, l'exposition Kunst in Deutschland, 1898-1973 propose à son tour de présenter un panorama de la scène artistique nationale, sur une plus longue durée. Sigmar Polke, Georg Baselitz et Markus Lüpertz sont toujours absents du catalogue ${ }^{(15)}$. Une seule œuvre de Gerhard Richter est présentée, dans la section qui présente l'année 1968, alors que l'artiste commence officiellement sa carrière en RFA en $1963^{(16)}$.

Comment expliquer cette sous-représentation, sur la scène artistique allemande des années 1970, de ces peintres qui apparaissent aujourd'hui comme les artistes allemands les plus renommés, alors qu'ils ont précisément commencé leur carrière dix ans auparavant? Ils pâtissent probablement de l'hégémonie de l'art américain sur la scène occidentale. Après le règne de l'art informel dans les années 1950, le Pop art semblait être le mouvement dans la direction de laquelle les artistes allemands (et européens) devaient s'inscrire pour être visibles, alors que dans les années 1970, le phénomène majeur qui s'était imposé aux États-Unis était celui de la dématérialisation de l'œuvre d'art, visant la remise en cause de l'objet-peinture et de l'objet-sculpture au profit de

14 En réalité, le nom de Sigmar Polke est bien évoqué lorsque l'auteur évoque le développement du «réalisme capitaliste» afin de présenter l'œuvre de Gerhard Richter. Toutefois, seules les œuvres de ce dernier bénéficient d'une double page de reproductions dans l'ouvrage. Voir Rolf-Gunter Dienst, Deutsche Kunst : eine neue Generation, Cologne, DuMont Schauberg, 1970, n. p.

15 Voir Kunst in Deutschland, 1898-1973, cat. exp., Hamburger Kunsthalle, Hambourg; Städtische Galerie - Lenbachhaus, Munich, 1973-1974, n. p.

16 Sur son internet officiel, Gerhard Richter a choisi de dater le début de sa carrière en 1962, avec l'œuvre Table. Voir www.gerhard-richter.com/fr/art/. 
pratiques artistiques privilégiant l'éphémère et/ou le multiple, comme la performance, le Land art, l'art conceptuel, etc. Ainsi, les artistes allemands qui développaient des pratiques au diapason de ces mouvements développés aux États-Unis, tel Joseph Beuys au sein de Fluxus, ont pu sembler dans les années 1970 plus pertinents que ceux qui développaient une peinture figurative qui n'était ni Pop, ni hyperréaliste, ni minimaliste - à l'instar de celle de Sigmar Polke, Georg Baselitz ou Lüpertz.

Le véritable tour de force de la scène allemande a été de réévaluer durablement et a posteriori ce passé pictural. Autrement dit, ce n'est que dans les années 1980 que l'œuvre des peintres allemands ayant commencé leur carrière dans les années 1960 a été mise sur un piédestal et qu'a été construit, sur cette base, un récit sur l'art contemporain allemand mettant l'accent sur la peinture - un récit au sein duquel la production picturale des années 1980 apparaîtrait comme la digne héritière de deux décennies de peinture. Sur un plan international, la décennie 1980 est marquée par une nouvelle affirmation de la peinture figurative, sous forme de scènes nationales souvent défendues comme telles (telles que la transavanguardia italienne, la bad painting américaine, la Figuration libre française et les Neuen Wilden allemands, c'est-à-dire les «nouveaux fauves»). Ce phénomène se présente comme une réaction contre la trop grande intellectualisation de l'art générée par toutes les tendances de la dématérialisation de l'œuvre d'art déjà évoquées ${ }^{(17)}$. Dans ce contexte et à la différence des autres pays, ce n'est pas seulement la jeune génération de peintres allemands (Werner Büttner, Walter Dahn, Martin Kippenberger, Helmut Middendorf, Albert Oehlen, Salomé, etc.) nés au début des années 1950 qui est mise sur le devant de la scène et associée à la vague néoexpressionniste observée ailleurs : les peintres nés aux alentours de la Seconde Guerre mondiale, comme Georg Baselitz, Anselm Kiefer ou encore Markus Lüpertz, sont eux aussi qualifiés de «nouveaux fauves». Dans ce contexte, cette expression "nouveaux fauves» devient le label allemand célébrant le renouveau de la peinture figurative, s'imposant comme un équivalent pratique des autres étiquettes célébrant la vitalité de la scène italienne, française et américaine.

Alors que ce retour en fanfare de la peinture déchire la scène artistique américaine, provoquant notamment l'écriture de quelques célèbres articles assassins dans les revues October ou Art in America attaquant ce médium présenté comme réactionnaire et complice du capitalisme ${ }^{(18)}$, le discours de défense de la peinture comme un médium contemporain se révèle en Allemagne être la stratégie idéale pour s'affranchir ostensiblement de la tutelle américaine. Il s'agit de refuser le discours mainstream sur la dématérialisation de l'art. Dès septembre 1981, le théoricien de l'art allemand Wolfgang Max Faust écrit ainsi dans la revue (anglophone) internationale Artforum:

«Après un art fait par et pour l'intellect [...], nous sommes maintenant témoins d'une redécouverte du dessin et de la peinture simples et spontanés. De prime abord, cela peut

17 Voir par exemple Suzanne Pagé, «Baroques 81 », Baroques 81, cat. exp., Paris, 1981, p. 7, ou encore Maurice Besset, «Les calculs de l'innocence» (L'Italie et l'Allemagne, cat. exp., Genève, 1983), L'Époque, la mode, la morale, la passion, cat. exp., Centre Pompidou, Paris, 1987, p. 568.

18 Voir par exemple Benjamin BuchloH, «Figures of Authority, Ciphers of Regression. Notes on the Return of Representation in European Painting», October, $\mathrm{n}^{\circ}$ 16, printemps 1981, p. 39-68, suivi de D. Crimp, "The End of Painting» (note 4); ou encore Craig Owens, "Back to the Studio", Art in America, 70/1, janvier 1982, p. 99-107. 
paraître à certains comme un retour nostalgique (ou même réactionnaire) à des traditions longtemps négligées; après un examen plus minutieux, l'on réalise qu'un changement est en train de prendre place et que ces artistes ont des positions esthétiques qui sont réellement neuves [...]. Que ceci apparaisse avec une véhémence particulière en Allemagne de l'Ouest [...] est dû en partie à la situation socio-culturelle générale de ce pays. Après la Seconde Guerre mondiale, en résultat de la politique nazie barbare, l'Allemagne n'avait pour ainsi dire pas d'art d'avant-garde et se tournait d'un courant international à un autre pour se nourrir. La France, puis les États-Unis et le renouveau d'anciennes phases de l'avant-garde internationale d'avant-guerre devinrent les points fixes autour desquels tournait l'art allemand occidental. [...] au moment où se terminaient les années 70, les artistes spécifiquement européens étaient à nouveau aux premiers rangs de la discussion d'avant-garde» ${ }^{(19)}$.

À l'issue de ce premier texte affirmant déjà une soif d'indépendance artistique à l'encontre des États-Unis, Wolfgang Max Faust publie en 1982 avec Gerd de Vries un ouvrage intitulé Hunger nach Bildern: deutsche Malerei der Gegenwart, qui propose une histoire de la peinture allemande contemporaine. Y est ainsi expliqué dès les premières pages que la nouvelle peinture allemande, sans être nationaliste, n'a pas de modèle étranger ${ }^{(20)}$ - quand bien même le retour à la peinture est un phénomène observé dans d'autres pays d'Europe et aux États-Unis. Sa particularité tiendrait à son indépendance par rapport à la situation artistique internationale.

Sur ce modèle se met en place progressivement une surprenante distorsion des récits passés. Les jeunes peintres allemands qui paraissaient inintéressants dans les années 1960-1970, car trop peu liés aux tendances artistiques internationales, deviennent progressivement les artistes les plus respectés des années 1980, et ce grâce à cette actualité artistique internationale de renouveau de la peinture figurative qui contribue à changer le regard sur leurs tableaux. Ils jouissent dès lors en RFA d'une image d'artistes rebelles qui, venus pour beaucoup d'Allemagne de l'Est ${ }^{(21)}$, auraient su défier la tendance générale, dictée par les États-Unis (le Pop, le minimalisme, puis l'art conceptuel, etc.).

\section{Un récit qui dépasse les seuls intérêts de la scène allemande}

Plusieurs articles, ouvrages et catalogues d'exposition des années 1980 adoptent ce même point de vue, contribuant ainsi à réécrire l'histoire de l'art allemand des dernières décennies. La peinture y est présentée comme le principal élément que la scène allemande ne partage avec aucune autre: elle serait constitutive d'une identité artistique nationale, et ce depuis les années 1960. Cet état de fait aurait été enfin révélé dans les années 1980, grâce à la fin du règne hégémonique des avant-gardes qui, depuis le début du siècle, défendaient l'idée que l'histoire avait un sens, une direction unique - une logique au nom de laquelle la scène américaine avait mis de côté le médium

19 Wolfgang Max FAust, «Les tendances dans l'art allemand contemporain» (Artforum, septembre 1981), L'Époque, la mode, la morale, la passion, cat. exp., Centre Pompidou, Paris, 1987, p. 581 sq.

20 Wolfgang Max FAust, Gerd de VRIEs, Hunger nach Bildern: deutsche Malerei der Gegenwart, Cologne, DuMont, 1982, p. 10.

21 Georg Baselitz (1938) par exemple a grandi en Saxe, près de Dresde; Gerhard Richter (1932) est né à Dresde, tout comme A.R. Penck (1939); Sigmar Polke (1941-2010) vient de Silésie, dans une région qui appartient aujourd'hui à la Pologne. 
peinture dans les années 1960-1970. Parmi les expositions allemandes qui entérinent ce nouveau récit sur l'histoire de l'art allemand contemporain, présentant sur un piédestal la peinture, figurent tout particulièrement trois manifestations: Westkunst, zeitgenössische Kunst seit 1939 organisée en 1981-1982 à Cologne, Zeitgeist à Berlin en 1982, puis Bilderstreit: Widerspruch, Einheit und Fragment in der Kunst seit 1960 qui a lieu dans la même ville en 1989. Ces trois expositions n'étaient pas tournées exclusivement vers la production artistique allemande - ce qui les rendit d'autant plus efficaces et redoutables. Elles présentaient un panorama de la création contemporaine, faisant la part belle aux artistes allemands, et notamment aux peintres, ce que ne manquèrent pas de souligner les critiques étrangers ${ }^{(22)}$.

Pourtant, il serait injuste et erroné de conclure hâtivement que la valorisation de la peinture contemporaine allemande à partir des années 1980 ait été façonnée et orchestrée exclusivement par des théoriciens de l'art, des conservateurs et des galeristes allemands soucieux de défendre au mieux les intérêts de la scène artistique de leur pays. En effet, un certain nombre de personnalités étrangères, européennes et américaines, ont participé à cette entreprise de réécriture de l'histoire de l'art contemporain allemand, présentant volontiers le choix du médium peinture comme un acte de résistance à l'impérialisme minimal et conceptuel américain. Par exemple, en 1989, était présentée à Düsseldorf et Francfort-sur-le-Main l'exposition Neue Figuration, deutsche Malerei, 1960-1988. À lui seul, ce titre précisait le propos de l'exposition, centrée sur une nouvelle peinture figurative allemande prenant sa source dans les années 1960. Il pouvait ainsi laisser croire à une opération d'autopromotion nationale de ce nouveau récit. Or, cette exposition fut conçue initialement aux États-Unis où elle fut d'abord présentée sous le titre Refigured Painting: The German Image 1960-88, avant de voyager à Toledo en Ohio, à New York (au Guggenheim Museum) et à Williamstown dans le Massachusetts.

Valoriser la peinture contemporaine allemande put devenir un cheval de bataille pour des individus n'ayant pas grandi ou pas travaillé en Allemagne à partir du moment où cette prise de position était un moyen de contester une vision téléologique trop rigide de l'histoire de l'art. Ainsi défendre la scène picturale allemande pouvait être motivé par l'ambition de défendre un autre type de production artistique que celle participant de la dématérialisation de l'œuvre d'art - et non par la seule volonté d'ébranler l'hégémonie américaine. Et cette situation de bouleversement historiographique, entérinant

22 Voir par exemple Christian BERNARD, «Westkunst, tournant des années 90 », art press, $\mathrm{n}^{\circ} 130$, novembre 1988, p. 15. À propos de la section «Heute» («Aujourd'hui») de l'exposition Westkunst, Christian Bernard, alors directeur de la Villa Arson à Nice, soulignait une «sélection où ne figurait aucun artiste français tandis que les USA s'y taillaient la part du lion (quatorze artistes), suivis par la RFA (huit), l'Italie (cinq), l'Autriche (quatre), les Pays-Bas (trois), la Suisse (deux) et le Canada (un) - soit 22 à 15 en faveur de l'Europe». Il notait que la décennie 1980 était celle de «la conquête du leadership européen par la RFA», scellant «la marginalisation accrue de la France». Voir également Catherine Millet, «Bilderstreit, l'après après-guerre», art press, n 137, juin 1989, p. 66. La critique soulignait une «inégalité flagrante des effectifs accordés aux factions de la Bilderstreit: une vingtaine de Baselitz contre quatre Ryman, par exemple» et déplorait la fin d'une époque «au cours de laquelle réaliser une grande exposition pour rassembler le public signifiait aussi rassembler les esprits autour d'œuvres qui méritent une attention particulière et de débats qui dépassent les rivalités commerciales et nationalistes». 
la fin des avant-gardes, trouvait une résonance aussi bien en Europe qu'aux États-Unis. Suivant cette logique, le conservateur britannique Norman Rosenthal présentait dès 1982 la peinture allemande, parce que figurative, comme la potentielle victime de l'hégémonie passée de l'abstraction américaine dans le catalogue de l'exposition Zeitgeist (Berlin, 1982), une manifestation importante qui contribua à promouvoir la peinture allemande contemporaine (en associant l'ancienne et la jeune génération):

«Pourquoi Brice Marden (né en 1938), dont l'œuvre repose sur l'importante tradition du constructivisme européen, passe pour un héraut de l'avant-garde, et pourquoi Georg Baselitz (né en 1938), qui se saisit de la puissante tradition de l'expressionnisme qu'il poursuit dans son œuvre, est présenté comme un épigone? - Manifestement parce que nous avons cru pendant longtemps, endoctrinés par une complexe idéologie artistique que la modernité et les avant-gardes de la dernière décennie devaient découler [...] de l'abstraction, du constructivisme ou peut-être un peu de Dada dans un extrême compromis » ${ }^{(23)}$.

Dans le même catalogue, l'Américain Hilton Kramer posait le même diagnostic, défendant plus largement la peinture figurative contre l'art abstrait, ayant selon lui profité trop longtemps d'un règne hégémonique sur l'histoire de l'art du $\mathrm{xx}^{\mathrm{e}}$ siècle ${ }^{(24)}$.

Ce discours était à nouveau sensible dans le catalogue de l'exposition German Art in the 20th century qui eut lieu à la Royal Academy de Londres en 1985, avant d'être présentée à la Staatsgalerie de Stuttgart en 1986. Le collectionneur allemand Günther Gercken y mettait en avant une peinture figurative indépendante qui, dès les années 1960, refusait «l'uniformité internationale» pour mieux renouer avec ses origines allemandes, en s'affranchissant par là même du «dogme d'un progrès linéaire», traçant notamment une ligne droite entre l'impressionnisme et l'art abstrait ${ }^{(25)}$. Il y ajoutait que «selon les anciens critères d'évaluation», la peinture allemande contemporaine était apparue «comme non avant-gardiste», qu’elle fut de ce fait «méprisée, car perçue comme un pas en arrière", alors qu'elle "s'avère désormais comme le pas en avant inattendu et imprévisible, dont les effets se laissent encore percevoir aujourd'hui et qui a conduit à une réévaluation de l'art du passé» ${ }^{(26)}$. Ce discours semble ainsi user des mêmes ressorts historiographiques que celui, passé, sur la dématérialisation de l'art: la peinture contemporaine allemande constituerait un progrès (Fortschritt). L'équipe organisant cette manifestation était composée du Grec Christos M. Joachimides, du Britannique Norman Rosenthal et de l'Autrichien Wieland Schmied. Avant de se retrouver pour organiser l'exposition Zeitgeist en 1982 à Berlin, les deux premiers commissaires s'étaient déjà fait remarquer par l'exposition A New Spirit in Painting à Londres en 1981, qui prit part au processus de promotion du retour à la peinture sans chercher à valoriser une scène nationale plus qu'une autre. Mais dans l'exposition de 1985, la peinture contemporaine allemande était bien seule au centre des attentions. Ce choix peut certes s'expliquer par le fait que le Grec Christos M. Joachimides s'était établi en Allemagne depuis les années 1950, y exerçant une activité de commissaire

23 Norman Rosenthal, Zeitgeist, cat. exp., Martin-Gropius-Bau, Berlin, 1982, p. 9.

24 Hilton Kramer, ibid., p. 16.

25 Günther Gercken, «Figurative Malerei nach 1960 », Deutsche Kunst im 20. Jahrhundert, Malerei und Plastik, 1905-1985, Londres, Royal Academy; Stuttgart, Staatsgalerie, 1985-1986, p. 468. 
d'exposition indépendant. Il n'en demeure pas moins que le choix de travailler, une nouvelle fois, en collaboration avec le Britannique Norman Rosenthal ne semble pas anodin, pas plus que le fait de présenter cette exposition sur la peinture allemande au $\mathrm{xx}^{\mathrm{e}}$ siècle, dans un premier temps, en Grande-Bretagne.

Cette implication de personnalités extérieures au monde de l'art allemand dans la promotion de la peinture allemande contemporaine permet de comprendre pourquoi ce discours de mise en avant de la peinture contemporaine allemande n'a pas été un épiphénomène englouti à la fin des années 1980 dans l'effondrement médiatique du retour à la peinture, en même temps que la carrière d'un certain nombre de jeunes peintres (toutes nationalités confondues), ayant profité une courte décennie d'un effet de mode. En effet, alors que le label «nouveaux fauves» se transformait déjà en souvenir confus d'un engouement pour une peinture libre et expressive, l'idée selon laquelle il existait une scène picturale solide et dynamique en Allemagne (depuis plusieurs décennies), elle, avait réussi à s'imposer dans les esprits.

C'est précisément après cette décennie, en 1990, qu'Isabelle Graw, une jeune critique d'art allemande, fonde à Cologne une nouvelle revue, Texte zur Kunst, sur le modèle de la célèbre revue américaine October, qui s'était illustrée dès sa création en 1976 pour ses attaques répétées contre le médium peinture et pour sa déconstruction des récits sur la modernité artistique véhiculés par le critique d'art américain Clement Greenberg ${ }^{(27)}$. Isabelle Graw partage avec ses collègues américains (Rosalind Krauss, Douglas Crimp, Craig Owens...) la même ambition théorique et les mêmes références (Clement Greenberg certes, mais aussi la fameuse "french theory» incarnée par Barthes, Deleuze, Derrida, Foucault, etc.). Toutefois, elle se démarque radicalement de ses homologues américains par sa volonté de défendre le médium peinture sur la scène actuelle, en démontrant sa contemporanéité. Ainsi, il n'est pas anodin de constater que son dernier ouvrage, paru en décembre 2017, s'intitule Die Liebe zur Malerei, Genealogie einer Sonderstellung et qu'il a déjà été traduit en anglais. La critique d'art allemande s'y intéresse, sans surprise, au médium peinture, à la place singulière qu'il occupe dans le paysage de l'art contemporain et à tous les éléments qui font sa contemporanéité. Ses exemples sont certes largement puisés dans la production allemande contemporaine, mais paraissent à égalité avec ceux issus de la production picturale américaine.

Cette volonté de défendre le médium peinture - qui s'observe ainsi à divers endroits en Allemagne depuis les années 1980 - s'avère aujourd'hui encore être un des éléments les plus remarquables, et le plus singulier, des discours qui soutiennent la production artistique contemporaine du pays. 


\section{Résumé}

Depuis plusieurs décennies, la peinture apparaît comme un médium privilégié sur la scène artistique allemande contemporaine. Cette situation s'est construite dans les années 1980: alors que la peinture figurative revient sur le devant de la scène en Europe comme aux États-Unis, l'Allemagne réussit à s'imposer comme le pays défenseur d'un médium qu'une grande partie de la scène artistique américaine s'était évertuée à présenter comme obsolète dans les années 1960-1970. Défendre la peinture apparaît alors comme une stratégie particulièrement efficace pour concurrencer les États-Unis, le principal pays prescripteur en matière d'art contemporain.

\section{Zusammenfassung}

Seit einigen Jahrzehnten erscheint die Malerei als ein bevorteiltes Medium der deutschen zeitgenössischen künstlerischen Szene. Diese Situation entstand in den achtziger Jahren. Als die figurative Malerei im Vordergrund in Europa sowie in den Vereinigten Staaten zurückkehrt, ist Deutschland das Land geworden, das ein Medium verteidigt, das von einem großen Teil der amerikanischen künstlerischen Szene in den sechziger und achtziger Jahren als obsolet betrachtet wird. Die Verteidigung der Malerei erscheint also als eine besonders effiziente Strategie, um die Vereinigten Staaten - das verschreibende Land was die Gegenwartskunst betrifft - zu konkurrieren. 